\title{
PERFORMANCE EVALUATION STUDY OF AN AVAILABLE BANDWIDTH MEASUREMENT TECHNIQUE IN MULTI-HOP WIRELESS AD HOC NETWORKS
}

\author{
Soumer Brahim, Kamoun Farouk, Tounsi Hajer \\ CRISTAL Laboratory ENSI, Manouba University \\ brahim.soumer@cristal.rnu.tn,.frk.kamoun@planet.tn, Hajer.Tounsi@cristal.rnu.tn
}

\begin{abstract}
In an ad hoc network, mobile nodes communicate with each other using multihop wireless links. There is no stationary infrastructure; for instance, there are no base stations. Each node in the network also acts as a router, forwarding data packets for other nodes. A central challenge in the design and evaluation of ad hoc networks is the estimation and the monitoring of network resources such as available bandwidth. Considering the dynamic routing protocols that can efficiently find routes between two communicating nodes, the high level of loss and the interference between node transmissions, the available bandwidth measurement techniques already provided by the literature may be inaccurate. Our goal is to carry out a systematic performance study of SloPS's [1] behavior when acting with three dynamic routing protocols for ad hoc networks: the Dynamic Source Routing protocol (DSR) [2,3], the Ad Hoc On-Demand Distance Vector protocol (AODV) $[4,5]$ and the Destination-Sequenced Distance Vector (DSDV) [6].
\end{abstract}

Keywords: measurement techniques, available bandwidth, ad hoc networks

\section{Introduction}

The research community is developing a set of metrics and techniques for bandwidth measurement. Many of them [7] are well understood and can provide accurate estimates under certain conditions. Network measurement techniques can be classified into two categories: passive measurement [8] and active probing [9] [10]. Passive measurement tools use the trace history of existing data transmission. While potentially very efficient and accurate, their scope is limited to network paths that have recently carried user traffic. Active probing, on the other hand, can explore the entire network. The packet pair technique [10] is one of the most popular active probing techniques. The basic idea of packet pairs is that the sender sends a pair of packets, which are echoed back by the destination. By measuring the changes in the packet spacing, the sender can estimate the bandwidth properties of the network path. While the packet pair mechanism is a reliable method for measuring the bottleneck link capacity of a network path [10], its use to measure the available bandwidth has had more mixed results. The capacity $\mathrm{C}$ of an end-to-end path is the maximum IP layer rate that the path can transfer from source to sink. In other words, 
the capacity of a path establishes an upper bound on the IP layer throughput that a user can expect to get from that path. The minimum link capacity in the path determines the end-to-end capacity $\mathrm{C}$. The hop with the minimum capacity is the narrow link on the path. We note that some layer-2 technologies do not operate with a constant transmission rate. For instance, IEEE $802.11 \mathrm{~b}$ wireless LANs transmit their frames at $11,5.5,2$, or $1 \mathrm{Mbps}$, depending on the bit error rate of the wireless medium. We can define this rate as the link capacity during time intervals in which the capacity remains constant. Another important metric is the available bandwidth of a link or end-to-end path. The available bandwidth of a link relates to the unused, or "spare", capacity of the link during a certain time period. So even though the capacity of a link depends on the underlying transmission technology and propagation medium, the available bandwidth of a link additionally depends on the traffic load at that link, and is typically a time-varying metric.At any specific instant in time, a link is either transmitting a packet at the full link capacity or it is idle, so the instantaneous utilization of a link can only be either 0 or 1.Previous measurement techniques performance comparisons [9][17] had demonstrated that Pathload [1] is the most accurate technique to estimate available bandwidth. In this study, we cover Pathload measurement technique as well as its behaviour in ad hoc network environment using simulation. We carry out a systematic performance study of SloPS's [1] behaviour when acting with three dynamic routing protocols for ad hoc networks: DSR [2,3], AODV [4,5] and DSDV [6].The paper is organized as follows. Section 2 gives a taxonomy of the related work in bandwidth estimation. The simulation parameters and scenarios are presented in section 3.1 while the various measurement techniques and methodology are illustrated in section 3.2. The simulation results are examined in section 4 and we conclude in section 5 .

\section{Related work}

\subsection{Available Bandwidth measurement techniques}

The Available bandwidth must be averaged over a reasonable time interval, so packet pair techniques often use packet trains. A typical example of an active measurement tool for available bandwidth is PBM (Packet Bunch Mode) [11]. If routers in the network implement fair queueing, the bandwidth indicated by the back-to-back packet probes is an accurate estimate of the "fair share" of the bottleneck link's bandwidth [12]. Another tool, cprobe [13], sends a short sequence of echo packets between two hosts. By assuming that "almost-fair" queueing occurs during the short packet sequence, cprobe provides an estimate for the available bandwidth along the path between the hosts. Treno [14] uses flow control and congestion control algorithms similar to those used by TCP to estimate available bandwidth. The work in [10] mentions a technique for estimating the available bandwidth based on the Asymptotic Dispersion Rate (ADR). Pathload [1] tool proposes to characterize the relationship between probing speed and available bandwidth by measuring the one way delay of probing packets. 


\subsection{SloPS (Self Loading Periodic Streams) : The Pathload technique}

SLoPS is a recent measurement methodology for measuring end-to-end available bandwidth[1]. The source sends a number $\mathrm{K}=100$ of equal-sized packets (a "periodic packet stream") to the receiver at a certain rate $\mathrm{R}$. The methodology involves monitoring variations of the one way delays of the probing packets. If the stream rate $R$ is greater than the path's available bandwidth $A$, the stream will cause a short term overload in the queue of the tight link. One way delays of the probing packets will keep increasing as each packet of the stream queues up at the tight link. On the other hand, if the stream rate $R$ is lower than the available bandwidth $\mathrm{A}$, the probing packets will go through the path without causing an increasing backlog at the tight link and their one way delays will not increase. One way delays increase only when the stream rate $R$ is larger than the available bandwidth A. In SLoPS the sender attempts to bring the stream rate $\mathrm{R}$ close to the available bandwidth $\mathrm{A}$, following an iterative algorithm. The sender probes the path with successive packet trains of different rates, while the receiver notifies the sender about the one-way delay trend of each stream. The available bandwidth estimate A may vary during the measurements. SLoPS detects such variations when it notices that the one-way delays of a stream do not show a clear increasing or non-increasing trend; (a grey region) which is related to the variation range during the measurements.

\section{Simulation study}

\subsection{Simulation scenarios and parameters}

The overall goal of our simulation experiments is to measure the ability of SloPS to resist to network topology change while continuing to successfully measure available bandwidth based on estimating the One Way Delay along the path. In this sense, our basic methodology is to apply to the network a variety of workloads in order to define the measurement techniques performance under some conditions. The measurement techniques evaluation is based on the simulation of 50 wireless nodes forming an ad hoc network, moving over a square flat space $(500 \mathrm{~m}-500 \mathrm{~m})$ during 900 seconds of simulated time. In order to enable fair comparisons of the routing protocols effect, it was critical to challenge SLoPS with identical loads and environmental conditions. Each run of the simulator accepts in input a scenario file that describes the exact motion of each node and the exact sequence of packets originated by each node, with the exact time at which each change in motion or packet origination is to occur. We choose our cross traffic sources to be constant bit rate (CBR) sources. Nodes in the simulation move according to the "random waypoint" model [3]. The movement scenario files that we used for each simulation are characterized by a pause time. Each node begins the simulation by remaining stationary for pause time seconds. It then selects a random destination in the $500 \mathrm{~m}-500 \mathrm{~m}$ space and moves to that destination at a speed, distributed uniformly between 0 and some maximum speeds. We have fixed two maximum speeds: $1 \mathrm{~m} / \mathrm{s}$ for low speed motion and $20 \mathrm{~m} / \mathrm{s}$ for high speed motion. Each simulation ran for 900 seconds of simulated time. We ran our simulations with movement patterns generated for 8 different pause times: $0,30,60,120,300,600,800$ and 
900 seconds. Because the performance of the measurement technique is very sensitive to movement pattern, we generated scenario files with 80 different movement patterns, 10 for each value of pause time. All three routing protocols were run on the same 80 movement patterns. We implemented SLoPS as an application object in NS (Network Simulator). Thus Pathload consists of two processes. The sender (SND) running at the server and the receiver process $(\mathrm{RCV})$. The control channel transfers messages regarding the characteristics of each stream (the abortion or end of the measurement process etc). We fixed the receive timeout as two seconds because we noticed that less than this value, the reactive routing protocols may not have already routes in their cache for our scenarios. In the other hand, this timer must not be very large because the execution time of all the process may increase and consequently Pathload can't converge before the route definition changes. Another important parameter is the acceptable loss. In fact when considering the analysis of the stream, Pathload ignore all the streams that present a loss more than a certain limit. We fixed the value of the acceptable loss as 0.25 because of the delivery ratio in low mobility conditions is higher than $75 \%$ for all the routing protocols [15].

\subsection{Error measurement methodology}

3.2.1 Metric. We define the relative measurement error of Pathload as where AB-pathload is the available bandwidth estimated by Pathload, ABsimu is the available bandwidth along the path given by NS traces, and Capacity is the bottleneck link capacity. This metric is measured for the three routing protocols to compare Pathload reliability for each one of them.

3.2.2 Collecting methodology. To measure the relative-error, we must know every variable in the formula. But we know that available bandwidth is a dynamic variable and it changes along the simulation, so we decided to execute Pathload algorithm every 100 seconds, this is a sufficient time for Pathload to terminate the measurement. We added also the number of hops aspect, that's why we choose randomly a path for each length. For example, before starting the simulation, we have by an internal mechanism all the one hop length paths between all nodes every 100 seconds interval and then we choose just one of them to experiment pathload along it. We repeat this process for two, three and four hops paths long and of course for the nine hundred seconds intervals of the total simulation time. We noticed that for almost all the routing protocols, there are no paths with more than four hops that still with stable length within the 100 seconds interval especially when the pause time is very little and the movement speed is $20 \mathrm{~m} / \mathrm{s}$. Once the path definition is fixed, we know all the intermediate nodes. We can trace all the packets that these nodes are forwarding even with other nodes of the map. So we can, for every 100 seconds intervals, measure the amount of data forwarded by each node and consequently in each link of the path. With this method we can have at each time interval the minimum available bandwidth along the path and also because we fix the capacity as the bottleneck link rate which is $1,2,5$ or $11 \mathrm{Mb}$. Thus we have for each scenario hop length an output file with nine relativ-error values and this for each routing protocol. As pathload gives us an interval for the $\mathrm{AB}$, we take its 
center as AB-pathload variable. Finally, the relative-error for each pause time scenario is $\sum_{i=1}^{9} \frac{\text { relative-error }}{9}$

\section{Simulation results}

\subsection{Available bandwidth measurement}

The first set of experiments uses differing numbers of pause time with a low mobility speed of $1 \mathrm{~m} / \mathrm{s}$ and 10 traffic sources. Fixing for example the pause time at 500s, the available bandwidth for DSR, DSDV and AODV measured by NS traces and Pathload technique are illustrated in Figure 1 for the $900 \mathrm{~s}$ measurement period.

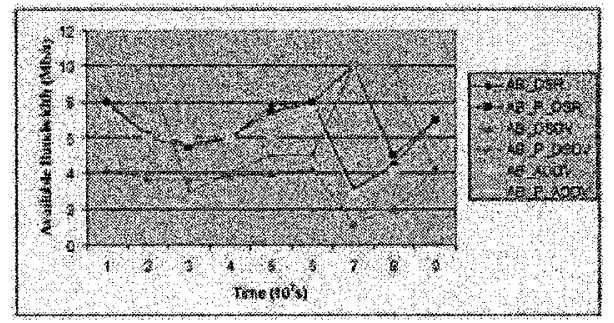

Figure 1. Available bandwidth for 1 Hop Pause $=500 \mathrm{~s} \mathrm{Speed}=1 \mathrm{~m} / \mathrm{s}$ sources $=10$

We call AB-P-x the available bandwidth measured by Pathload and AB-x the available bandwidth measured by NS traces and this when $\mathrm{x}$ is the routing protocol.First, we must notice that DSR and AODV outperforms DSDV in the real available bandwidth value performance during almost all measurement period. This is due to the low delivery percentage of DSDV comparing to the two other routing protocols especially for this pause time mobility (500s) [15].Figures 2 and 3 highlight the relative-error performance of Pathload when acting with the three routing protocols on our traffic loads of 10 sources. We consider in figure 2 the case of one hop routes and in figure 3 the case of two hops routes. The figure 1 presents the details of our measurements for the first case, using always the same method to compute relative-error for each 100 seconds interval. We notice in this figure that we measure first for each one hop route the available bandwidth using Pathload then we apply the technique described above to measure manually using NS traces the utilization of each link and then obtain the real value of the available bandwidth. Using a pause time equal to $500 \mathrm{~s}$, we can notice that we can have three main cases.

The first case is when Pathload can converge with a good performance and the relative-error is less than $10 \%$ for the three routing protocols (between $300 \mathrm{sec}$ and $600 \mathrm{sec}$ when the relative-error is $3 \%, 10 \%$ and $8 \%$ for respectively DSR, DSDV and AODV).The second case is when all the routing protocols give a bad performance (between $600 \mathrm{sec}$ and $700 \mathrm{sec}$ ). This is because Pathload can't converge due to cross traffic that increases the delivery time of Pathload Packet trains and consequently obliges the algorithm to ignore many times the same stream. In fact, Pathload uses a TCP connection to control 


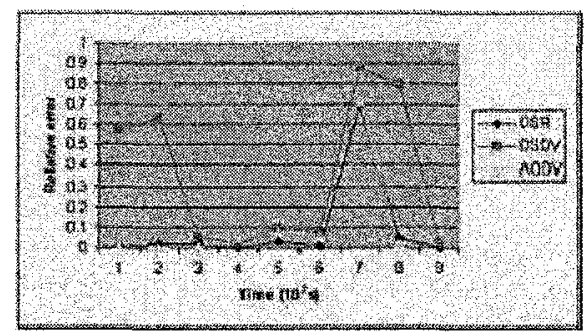

Figure 2. Relative-error for 1 Hop Pause $=500$ s Speed $=1 \mathrm{~m} / \mathrm{s}$ sources $=10$

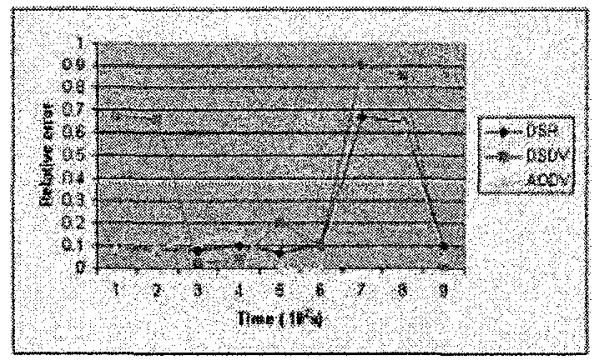

Figure 3. Relative-error for 2 Hops Pause $=500 \mathrm{~s}$ Speed $=1 \mathrm{~m} / \mathrm{s}$ sources $=10$

and validate each stream before calculating the heuristics metrics and decide the increasing trend of the One Way delay (OWD) ${ }^{1}$. This case can exist when the routing protocol packet delivery percentage is very low due to mobility or when the link utilisation is very high due to cross traffic. The last case is when Pathload gives good performance with one or more routing protocols and fail to converge with one other. This is for example the case of DSDV between 0 and $200 \mathrm{sec}$ and also between 700 and $800 \mathrm{sec}$. This case is due to the routing protocol proactive nature. In fact, in these time intervals DSDV could not find any route between the chosen pair of nodes or take a long time to complete the exhaustive list of routes between all nodes and this especially in the simulation starting. To evaluate the impact of hop length over Pathload performance, we fix the same sources for our one hop and two hops chosen paths (we must choose of course paths that have to still stable during the measurement $100 \mathrm{~s}$ period and this at least for two hops). This hypothesis is only for this experiment need and we keep the random paths selection for all the other experiments. We remark that more the path length grows more the Pathload accuracy decreases. In fact for the same time period we have for example between 0 and $100 \mathrm{sec}-$ onds the relative error is $1 \%, 57 \%$ and $1 \%$ for one hop path and $9 \%, 67 \%$ and $9 \%$ for two hopspath and this respectively for DSR, DSDV and AODV routing protocols. This increase is more important when the path utilizationis greater.

\footnotetext{
'When Pathload can't converge we fix AB-Pathload as $10 \mathrm{Mb}$ which is the initial rate value.
} 
We can take the [700-800] seconds interval when the relative error is $5 \%, 80 \%$ and $2 \%$ for one hop path and $65 \%, 85 \%$ and $64 \%$ for two hops path and this respectively for DSR, DSDV and AODV routing protocols.

\subsection{The mobility effects}

The next set of experiments (Figure 4) demonstrate the effect of mobility. We choose the average relative-error value calculated for different pause times and for all the four path length cases of one, two, three and four hops. As expected, the relative-error metric is converging to 1 when there is a very high node motion. For example, figure 4-a shows this metric as a function of both node mobility rate (pause time) and the routing protocols. For DSR and AODV, packet delivery ratio is between $95 \%$ and $100 \%$ of the packets in all cases that's why all Pathload packet trains are validated and a very little number of streams are ignored. DSDV fails to converge below pause time 300, where it delivers about $92 \%$ of its packets. At higher rates of mobility (lower pause times), DSDV does poorly, dropping to a $70 \%$ packet delivery alternate routes. Nearly all of the dropped packets are lost because a stale routing table entry directed them to be forwarded over a broken link. In fact, DSDV maintains only one route per destination and consequently, each packet that the MAC layer is unable to deliveris dropped since there are no alternate route. Figures 4b, 4-c and 4-d show that more the path length increases more the relative-error is greater. This is due to the cross traffic which is more important when the path contains larger number of hops. Also, the probability to have a path definition changes during Pathload execution time is more important when the number of hops in the path grows. In fact, we abort the measurement operation when we detect that a routing changes have occurred even when we have another route between the same source and destination. In summary, for low mobility and

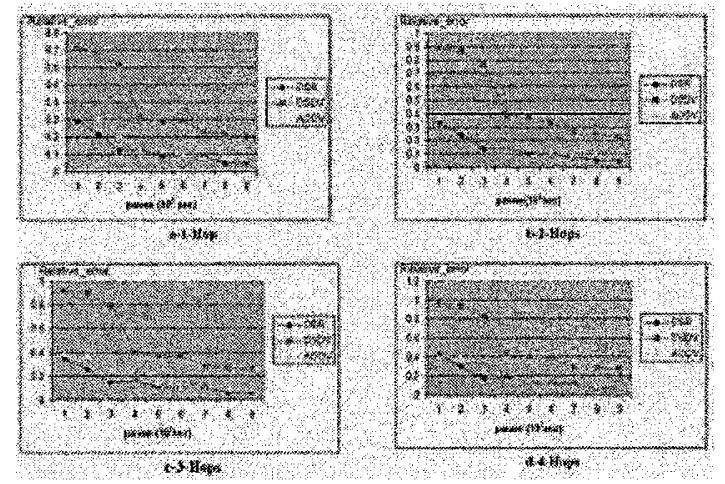

Figure 4. Relative error $=\mathrm{f}($ pause-time) when $\mathrm{Speed}=1 \mathrm{~m} / \mathrm{s}$ sources $=10$

with 10 sources of cross traffic, pathload can have an accuracy of $90 \%$ for DSR and AODV and of $70 \%$ for DSDV. For higher mobility (pause time $<300 \mathrm{~s}$ ), pathload is not the accurate tool. In fact, its relative error is greater than $40 \%$ for DSR and AODV and $90 \%$ for DSDV. 

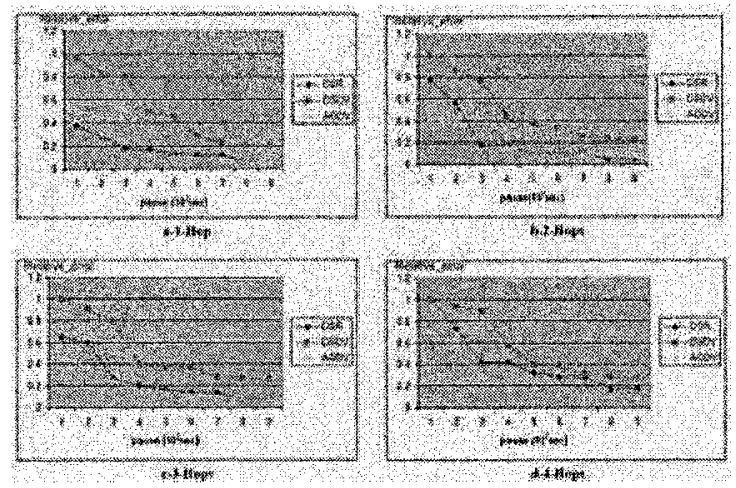

Figure 5. Relative error $=\mathrm{f}($ pause-time $)$ when $\mathrm{Speed}=1 \mathrm{~m} / \mathrm{s}$ sources $=30$

\subsection{Cross traffic effects}

In figure 5, we have 30 sources of cross traffic. In this experiment, we have noticed that DSR expresses more overhead to find routes. This is because DSR put all the route definition in the packet header of its route request messages and consequently the links bandwidth utilization is very important. Comparing between figure 4-a and 5-a results, we find that when there is no pause time, the relative-error has increased from $30 \%$ to $40 \%$ for DSR and AODV and from $70 \%$ to $95 \%$ for DSDV. For longer paths (figures 5-b,5-c and 5-d), the pathload performance degrades with DSR and high mobility conditions. In fact, the relative error of Pathload is greater than $60 \%$ for pause time $<200$ s. With AODV, Pathload stills have good performance (less than $40 \%$ for high mobility and one, two and three hops). This is not the case of four hops when the relative-error of Pathload is $70 \%$ and $57 \%$ respectively when the pause time $=0 \mathrm{~s}$ and $100 \mathrm{~s}$.

\subsection{The mobility speed effects}

In order to explore how the Pathload measurement technique scales with the change of the topology, we changed the maximum node speed from $1 \mathrm{~m} / \mathrm{s}$ to $20 \mathrm{~m} / \mathrm{s}$ and re-evaluated it with all three protocols over scenario files using this lower movement speed In this experience, a general remark is that many measurement tentatives fail because the routing changes occur during the measurement operation. In fact, we started the Pathload process each $100 \mathrm{sec}$ and we have noticed that among the 360 measurement tentative that we have done for each experience (each experience is repeated ten times with 9 values for each hop count) 161,152 and 201 routing changes during the measurement operation and this respectively for DSDV, DSR and AODV have been occurred. So these routing changes increase when the mobility speed increases and they are the principal source of error of the Pathload measurement technique. In fact, when the speed is $1 \mathrm{~m} / \mathrm{s}$ we have only 71,64 and 82 routing changes during the measurement execution for respectively DSDV, DSR and AODV. In figure 


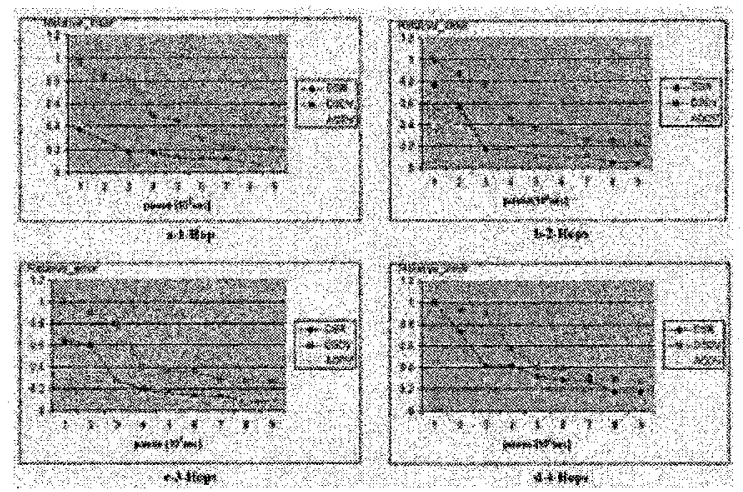

Figure 6. Relative error $=\mathrm{f}($ pause-time $)$ when $\mathrm{Speed}=20 \mathrm{~m} / \mathrm{s}$ sources $=10$

6 , we fix the mobility speed to $20 \mathrm{~m} / \mathrm{s}$ and we keep all the other conditions as paragraph 3.2. We notice that the performance of Pathload degrades with almost all the routing protocols, especially for DSR and AODV in comparison with their results when the mobility speed is $1 \mathrm{~m} / \mathrm{s}$. The difference between DSR and AODV, however, has grown from a factor of 1.2 to nearly a factor of 1.6 for four hops paths because DSR's caching is even more effective at lower speeds where the cached information goes stale more slowly. Although, due to its largely periodic nature, pathload with DSDV continues to have a constant relative-error as the case of the mobility speed equals to $1 \mathrm{~m} / \mathrm{s}$.

\section{Conclusions}

The area of ad hoc networking has been receiving increasing attention among researchers in recent years. Over the past few years, a variety of new available bandwidth measurement techniques targeted specifically at the ad hoc networking environment have been proposed, but little performance information on each technique and no detailed performance study of these techniques has previously been available in ad hoc network environment. In this work, we describe first SloPS technique and its integration into the ns network simulator. This new simulation environment provides a powerful tool for re-evaluating this technique and its behavior when operating in an ad hoc network. Then, we present the results of simulation comparing three multi-hop wireless ad hoc network routing protocols DSDV, DSR, and AODV. We present the results for a range of node mobility rates and movement speeds. This technique performs well in some cases yet has certain drawbacks in others. With DSDV, SloPS performs quite well, with an acceptable error-rate when node mobility rate and movement speed are low, and doesn't converge when node mobility increases. The performance of SloPS in presence of DSR was good at all mobility rates, however the use of source routing increases the number of routing overhead bytes required by the protocol so the measurement relative-error increases when movement speeds increases or the cross traffic is very large. Finally, Slops in presence of AODV performs almost as well as with DSR 
at almost all mobility rates and movement speeds and accomplishes its goal of eliminating source routing overhead. However, topology changes can occur during the measurement process which can lead Pathload packets train to switch to another path and consequently available bandwidth estimation will not be accurate especially when movement speed is $20 \mathrm{~m} / \mathrm{s}$.Many extensions can be applied to this study. It is obvious that this is not a complete study of all the major measurement techniques. In fact, other available bandwidth measurement techniques implementation in NS like IGI [16] or cprobe [13] can be added to the performance comparison. In addition, we believe that we must have a real integrated wireless ad hoc testbed that supports the different routing protocols and also ad hoc networks adapted monitoring techniques to collect real actives available bandwidth measurement techniques information's. This testbed is useful to validate our future measurement techniques performance studies and also the mobility impact over their behavior.

\section{References}

[1] Manish Jain and Constantinos Dovrolis. Pathload: A measurement tool for end-to-end available bandwidth. In Passive and Active Measurements, Fort Collins CO, March 2002.

[2] J.Broch, D. Johnson, and D. Maltz. The Dynamic Source Routing Protocol for Mobile Ad Hoc Networks, http://www.ietf.org/internet-drafts/draft-ietfmanet-dsr-03.txt, IETF Internet draft, Oct. 1999, work in progress.

[3] D. Johnson and D. Maltz. Dynamic Source Routing in Ad Hoc Wireless Networks. T. Imielinski and H. Korth, Eds. Mobile Computing, Ch. 5, Kluwer, 1996.

[4] C. E. Perkins and E. M. Royer, Ad Hoc On-demand Distance Vector Routing. Proc. 2nd IEEE Wksp. Mobile Comp. Sys. and Apps., Feb. 1999, pp. 90-100.

[5] C. E. Perkins, E. M. Royer, and S. R. Das, Ad Hoc on Demand Distance Vector (AODV) Routing http://www.ietf.org/internet-drafts/draft-ietfmanet- aodv-06.txt, IETF Internet Draft, July 2000 , work in progress.

[6] Charles E. Perkins and Pravin Bhagwat. Highly dynamic Destination-Sequenced DistanceVector routing (DSDV) for mobile computers. In Proceedings of the SIGCOMM '94 Conference on Communications Architectures, Protocols and Applications,

[7] Prasad, R.S., Murray, M., Dovrolis, C., Clay, K Bandwidth estimation: metrics, measurement techniques, and tools. IEEE Network Magazine (2003)

[8] S. Seshan, M. Stemm, and R. H. Katz SPAND: shared passive network performance discovery. in In Proc 1st Usenix Symposium on Internet Technologies and Systems

[9] Federico Montesino-Pouzols Instituto de Microelectrnica de Sevilla (IMSE-CNM) Seville, Spain Federico.Montesino@imse.cnm.es Comparative Analysis of Active Bandwidth Estimation Tools

[10] C. Dovrolis, P. Ramanathan, and D. Moore, What do packet dispersion techniques measure? in Proc. the Conference on Computer Communication (IEEE Infocom), Anchorage, Alaska, USA, Apr. 2001, pp. 905-914.

[11] Vern Paxson. Measurements and Analysis of End-to-End Internet Dynamics.. PhD thesis, U.C. Berkeley, May 1996.

[12] Srinivasan Keshav. Packet pair flow control. IEEE/ACM Transactions on Networking, February 1995.

[13] Robert L. Carter and Mark E. Crovella. Measuring bottleneck link speed in packet-switched networks. Technical report, Boston University Computer Science Department,

[14] Matthew Mathis and Jamshid Mahdavi. Diagnosing internet congestion with a transport layer performance tool. . In Proc. INET'96, Montreal, Canada, June 1996.

[15] Josh Broch David A. Maltz David B. Johnson Yih-Chun Hu Jorjeta Jetcheva A Performance Comparison of Multi-Hop Wireless Ad Hoc Network Routing Protocols.

[16] Ningning Hu, Student Member, IEEE, Peter Steenkiste, Senior Member, IEEE Evaluation and Characterization of Available Bandwidth Probing Techniques.

[17] Soumer B. Measurement techniques in wired and wireless heterogeneous environments. Cristal Laboratory ENSI Tunisia Master report Juin 2004. 